\title{
Quantitative Risk Assessment for Crude Oil Pipelines
}

\author{
Huseyin Murat Cekirge \\ Department of Mechanical Engineering, Prince Mohamed Bin Fahd University, Al Khobar, KSA
}

\section{Email address:}

hmcekirge@usa.net

\section{To cite this article:}

Huseyin Murat Cekirge. Quantitative Risk Assessment for Crude Oil Pipelines. International Journal of Environmental Monitoring and Analysis. Vol. 3, No. 3, 2015, pp. 147-153. doi: 10.11648/j.ijema.20150303.16

\begin{abstract}
Risk Assessment is an extremely useful tool in providing a framework in which to identify the possible hazards and evaluate the risks associated with all crude oil pipelines. By using index method and multivariable analysis, a methodology of the threat and risk of crude oil pipelines to environment are presented. General concepts are introduced and explained in detail. Principles of the methodology, the specific equations, and data required to prepare a risk analysis for environmental risk and failure analysis are discussed and explained, and an example is presented to illustrate the method.
\end{abstract}

Keywords: Crude Oil Pipelines, Risk Analysis, Risk Assessment, Index Method

\section{Introduction}

Risk Management in its broadest sense represents the successful control of all threats of harm and loss to a crude oil pipeline. The assessment and analysis produces numerical value of the risk involved and evaluates the results against specified risk criteria. The purpose of the Risk Analysis stage is to obtain an idea of the size or the scale of the risk, [1].

Simply, a risk assessment is finding out what could cause harm to people, environment, task or equipment. The results presents if it is being done enough, or need to do more to protect the installation. If risk is expressed in terms of High, Medium or Low, that qualitative. Attempt to express risk in numerical terms will be based on calculation using data for failure rates of equipment, human error, etc., that is $\operatorname{Low}(2$ or less), Medium(3-6) and High(6 or more).

These principles presented are specifically for crude oil pipelines in the context of this paper. A fundamental aim of this paper is to present an assessment method that is providing reasonable risk estimates for policy decisions. Certain assumptions used are part of the paper. The methodologies presented in the paper cover pipelines carrying crude oil.

The data required for a risk analysis includes pipeline data and site data. Some information that would aid in a risk analysis is proprietary to the pipeline operator. In general, the required data include:

- The location of the proposed pipeline site, including roads and major terrain feature boundaries;

- The location of the pipeline with respect to the proposed site, and specifically the segment lying within the site;
- Land use and terrain characteristics adjacent to and within the site;

A phase of environmental assessment study will sometimes have identified hazardous material pipelines near the site and several key characteristics of a pipeline such as:

- Location;

- The product transported;

- Diameter;

- Operating pressure;

- Materials of construction; and

- Date of construction.

The fundamental approach in the paper is the former, as described in detail in the remainder of this section. The paper also describes the latter, which can be done through the basic process by iterating on distance as described briefly later in this section.

\section{Risk Assessment System Features}

This document contains the results of the risk analysis technological pipeline transport system for which a methodology has been applied to meet new requirements and risk management infrastructure. It has been estimated as a basis for risk valorization and as a tool for the determination of the most dangerous areas in the event of a leak or spill which has not been declared a threatening even to foil spills to take appropriate action. Through the methodology used, is to also have a basis for risk management, represented a risk value for each section of the pipeline, and defined from the threat related variables, the constructive aspects of the system and 
the events that may originate from third parties or natural phenomenon. The study explains step by step the aspects of the methodology it is necessary that the operating group responsible for maintaining the Contingency Planning formation make necessary modifications to update the risk analysis, and also use these results in decisions for annual investment programs, conditioning it to risk management in the system.

This study initially present general aspect of risk, to facilitate understanding of the procedures and results of the methodologies employed in selection estimation causes and risk. Such methodologies are described later and are carried thereon to application pipeline transport system. The valuation methodology is based on the universal concept of risk, in which the parameters are determined amending it so semi quantitative leveraging estimation consequences (quantitative values) and qualitative assessments that define some parameters or aspects of risk.

\section{Risk Assessment Methodology}

The methodology used is a adaptation Indexing method developed in Pipeline Risk Management Manual, [2], [3], [4], and [5] using influence distances reported for the estimation of consequences and characteristics of operation pipeline transport system, to modify the risk variables, balance from the point of view of individual risk covered by Guidelines for Chemical Process Quantitative Risk Analysis that includes advances in Chemical Process Quantitative Risk Analysis (CPQRA), [6], [7]. [8] and [9].The methodology incorporates risk valorization specific data input and output to be updated constantly by staff in each infrastructure. It is the input characteristics that modify the risk, which are related to infrastructure, operation, maintenance, environment, prevention activities, and the characteristics of the load. These characteristics influence the frequency of a novice event, the probability of occurrence of a threatening event, the probability of producing damage in the area in which the event occurred given the same conditions and environmental vulnerability. These characteristics will be valued for the conditions of the pipeline transport system by sector, so that each will produce a separate segmentation along the pipeline. The combination of the values of these characteristics affected by factors defined for each weight results as a result a risk factor for the sector, which will be represented along a graphical pipeline to facilitate analysis. The methodology allows the calculation of a risk value of all infrastructures per unit length, besides being able to view the areas of greatest risk in the pipeline.

The risk is defined as the combination of four factors, one of which is a frequency (occurrences per unit time) and others are likely to occurrence (dimensionless values) of events that are chained in a threatening event, resulting in a risk value in terms of the occurrence of a particular damage per unit time. To facilitate risk management defines a set of characteristics that infer in the same and related infrastructure, ancillary systems, the operation, safety programs and environmental characteristics. To perform valorization should identify partitions that each feature occurs along the pipe and so to assign the respective values of each sector using sectorization maxima occur in the system. Each feature referred to faith will be given a relative value, which is a number between 0 and 10 following the guidelines presented in Table 1 and considering each risk characteristic independently.

\subsection{Characteristics of Risk Factors}

Threatening events considered are oil spills. If given an escape, not have any of these events, it is said that the event is a consequent pollution oil scattering or dispersion of the spill and oil. The environmental risk factors $\mathrm{Ci}^{\mathrm{i}} \mathrm{s}$ are listed in the following table:

Table 1. Environmental risk elements.

\begin{tabular}{lll}
\hline Variable & Definition & Explanation \\
\hline C1 & Surface Water Sensitivity & Very High $=10$, High $=7$, Medium $=5$, Low $=3$ and Not Sensitive $=1$ \\
& & Weight $=1$ \\
C2 & Very High $=10$, High $=7$, Medium $=5$, Low $=3$ and Not Sensitive $=1$ \\
& & Weight $=1$ \\
C3 & Terrestrial Ecological Resource & Very High $=10$, High=7, Medium $=5$, Low $=3$ and Not Sensitive $=1$ \\
& & Weight $=0.75$ \\
C4 & Land Use & Very High $=10$, High $=7$, Medium $=5$, Low $=3$ and Not Sensitive $=1$ \\
& & Weight $=0.75$ \\
C5 & Archaeology & Very High $=10$, High $=7$, Medium $=5$, Low $=3$ and Not Sensitive $=1$ \\
\end{tabular}

As base of four, the risks are classified as Not Sensitive $=0$, Low $=2$, Medium $=2$, High $=3$ and Very High $=4$.

To perform valorization should identify partitions that each feature occurs along the pipe and so to assign the respective values of each sector. The pipeline is beginning segmented for by one kilometer segmented. In the tables KP (Kilometer
Point) represents segments, and KP 5 presents segment starts at 4 th kilometer and ends at 5 th kilometer from staring point of the pipeline. The length of the segment may be chosen more and less than one kilometer. 
Each feature referred to faith will be given a relative value, which is a number between 0 and 10 following the guidelines presented in Table 1 and considering each risk characteristic independently.

Table 2. Qualitative values of environmental risk for the segments.

\begin{tabular}{|c|c|c|c|c|c|}
\hline $\mathbf{K P}(\mathbf{k m})$ & Surface Water Sensitivity & Ground Water Sensitivity & Terrestrial Ecological Resource & Land Use & Archaeology \\
\hline \multicolumn{6}{|l|}{0} \\
\hline 1 & Not Sensitive & Not Sensitive & Very High & Not Sensitive & Not Sensitive \\
\hline 2 & Not Sensitive & Not Sensitive & Very High & Medium & Not Sensitive \\
\hline 3 & Not Sensitive & Not Sensitive & Very High & Medium & Not Sensitive \\
\hline 4 & Low & Not Sensitive & Very High & Medium & Not Sensitive \\
\hline 5 & Not Sensitive & Not Sensitive & Very High & Medium & Not Sensitive \\
\hline 6 & Not Sensitive & Not Sensitive & Very High & Medium & Not Sensitive \\
\hline 7 & Not Sensitive & Not Sensitive & Very High & Not Sensitive & Not Sensitive \\
\hline 8 & Not Sensitive & Not Sensitive & Very High & Not Sensitive & Not Sensitive \\
\hline
\end{tabular}

Table 3. Quantitative values of environmental risk for the segments.

\begin{tabular}{|c|c|c|c|c|c|}
\hline KP (km) & Surface Water Sensitivity & Ground Water Sensitivity & Terrestrial Ecological Resource & Land Use & Archaeology \\
\hline \multicolumn{6}{|c|}{0} \\
\hline 1 & 1 & 1 & 10 & 1 & 1 \\
\hline 2 & 1 & 1 & 10 & 5 & 1 \\
\hline 3 & 1 & 1 & 10 & 5 & 1 \\
\hline 4 & 3 & 1 & 10 & 5 & 1 \\
\hline 5 & 1 & 1 & 10 & 5 & 1 \\
\hline 6 & 1 & 1 & 10 & 5 & 1 \\
\hline 7 & 1 & 1 & 10 & 1 & 1 \\
\hline 8 & 1 & 1 & 10 & 1 & 1 \\
\hline
\end{tabular}

Table 4. Normalization of environmental risk for the segments.

\begin{tabular}{|c|c|c|c|c|c|}
\hline KP (km) & Surface Water Sensitivity & Ground Water Sensitivity & Terrestrial Ecological Resource & Land Use & Archaeology \\
\hline $\begin{array}{l}\text { Weight } \\
0\end{array}$ & 1 & 1 & 0.75 & 0.25 & 0.25 \\
\hline 1 & 0.1 & 0.1 & 0.75 & 0.025 & 0.025 \\
\hline 2 & 0.1 & 0.1 & 0.75 & 0.125 & 0.025 \\
\hline 3 & 0.1 & 0.1 & 0.75 & 0.125 & 0.025 \\
\hline 4 & 0.3 & 0.1 & 0.75 & 0.125 & 0.025 \\
\hline 5 & 0.1 & 0.1 & 0.75 & 0.125 & 0.025 \\
\hline 6 & 0.1 & 0.1 & 0.75 & 0.125 & 0.025 \\
\hline 7 & 0.1 & 0.1 & 0.75 & 0.025 & 0.025 \\
\hline 8 & 0.1 & 0.1 & 0.75 & 0.025 & 0.025 \\
\hline
\end{tabular}

Table 5. Probability of environmental risk for the segments.

\begin{tabular}{|c|c|c|c|c|c|c|c|c|}
\hline To (km) & $\begin{array}{l}\text { Surface Water } \\
\text { Sensitivity }\end{array}$ & $\begin{array}{l}\text { Ground Water } \\
\text { Sensitivity }\end{array}$ & $\begin{array}{l}\text { Terrestrial Ecological } \\
\text { Resource }\end{array}$ & $\begin{array}{l}\text { Land } \\
\text { Use } \\
\end{array}$ & Archaeology & $\begin{array}{l}\text { Max } \\
\text { Probability }\end{array}$ & $\begin{array}{l}\text { Normalized } \\
\text { Probability } \\
\end{array}$ & $\begin{array}{l}\text { Total } \\
\text { Index }\end{array}$ \\
\hline \multicolumn{9}{|l|}{0} \\
\hline 1 & 0.4 & 0.4 & 3 & 0.1 & 0.1 & 0.75 & 3 & 1 \\
\hline 2 & 0.4 & 0.4 & 3 & 0.5 & 0.1 & 0.75 & 3 & 1.1 \\
\hline 3 & 0.4 & 0.4 & 3 & 0.5 & 0.1 & 0.75 & 3 & 1.1 \\
\hline 4 & 1.2 & 0.4 & 3 & 0.5 & 0.1 & 0.75 & 3 & 1.3 \\
\hline 5 & 0.4 & 0.4 & 3 & 0.5 & 0.1 & 0.75 & 3 & 1.1 \\
\hline 6 & 0.4 & 0.4 & 3 & 0.5 & 0.1 & 0.75 & 3 & 1.1 \\
\hline 7 & 0.4 & 0.4 & 3 & 0.1 & 0.1 & 0.75 & 3 & 1 \\
\hline 8 & 0.4 & 0.4 & 3 & 0.1 & 0.1 & 0.75 & 3 & 1 \\
\hline
\end{tabular}

\subsection{Base Frequencies and Modification Factors}

The frequency of an event is the expected number of times per length of pipe that an event will occur in a year. As an illustration, the excavation damage frequency for a given segment might be $1.4 \times 10-6$ based on historical incident data. That frequency represents the number of times that excavation is expected to cause a leak in that segment of the pipe in a year. For each segment of the pipeline, the frequency of events (and thus possible leaks) was determined by first assessing the frequency of each spill case individually, distributed among the three hole sizes. These were summed to give the total leak frequency, considering additivity, $\mathrm{i}=\mathrm{i}^{\text {th }}$ frequency,

$$
f=\sum_{i=1}^{n} f_{i}
$$

$\mathrm{fi}=$ leak event of $\mathrm{i}$,

$\mathrm{n}=$ number of leaks or 


$$
f=f_{m o}+f_{c o}+f_{i h}+f_{a i}+f_{n h},
$$

where:

$f_{m o}=$ leak frequency from material mechanical and operational faults,

$f_{c o}=$ leak frequency from corrosion,

$f_{i h}=$ leak frequency from intentional hostile action,

$f_{a i}=$ leak frequency from accidental / incidental action,

$f_{n h}=$ leak frequency from natural hazards.

The individual frequencies were determined by applying modification factors to a base leak frequency for each spill cause. The specific modification factors and hole size distributions are discussed for each of the relevant causes in the following subsections. The sizes are, Table 6:

Table 6. Hole sizes.

\begin{tabular}{lll}
\hline LEAK & HOLE & RUPTURE \\
\hline Leak Area $\left(\mathrm{cm}^{2}\right)$ & Leak Area $\left(\mathrm{cm}^{2}\right)$ & Leak Area $\left(\mathrm{cm}^{2}\right)$ \\
0.2 & 20.0 & full bore \\
\hline
\end{tabular}

The frequencies are presented by Tables 7, 8, 9, 10 and 11 .

Table 7. Frequency values of various events, [10], [11], [12], [13], [14], [15], [16] and [17].

\begin{tabular}{|c|c|c|c|c|c|c|c|c|c|}
\hline \multicolumn{4}{|c|}{ RAW FREQUENCY DATA } & \multicolumn{3}{|c|}{ SPLIT FRACTIONS } & \multicolumn{3}{|c|}{ FREQ FOR APPLICATION } \\
\hline CATEGORY & CAUSE & APPLICATION & $\begin{array}{l}\text { RAW } \\
\text { FREQ } \\
{[/ y \mathrm{~km}]}\end{array}$ & LEAK & HOLE & RUPTURE & LEAK & HOLE & RUPTURE \\
\hline MECH\& OP FAULTS & $\begin{array}{l}\text { Mechanical (Line } \\
\text { Pipe Fault) }\end{array}$ & General & $8.44 \mathrm{E}-05$ & 0.7 & 0.24 & 0.06 & $5.91 \mathrm{E}-05$ & 2.03E-05 & $5.06 \mathrm{E}-06$ \\
\hline & Op Faults & General & 4.78E-05 & 0.75 & 0.25 & 0 & $3.59 \mathrm{E}-05$ & $1.20 \mathrm{E}-05$ & $0.00 \mathrm{E}+00$ \\
\hline CORROSION & $\begin{array}{l}\text { Internal } \\
\text { External }\end{array}$ & $\begin{array}{l}\text { General } \\
\text { General }\end{array}$ & $\begin{array}{l}4.22 \mathrm{E}-05 \\
5.35 \mathrm{E}-05\end{array}$ & $\begin{array}{l}0.9 \\
0.9\end{array}$ & $\begin{array}{l}0.09 \\
0.09\end{array}$ & $\begin{array}{l}0.01 \\
0.01\end{array}$ & $\begin{array}{l}3.80 \mathrm{E}-05 \\
4.82 \mathrm{E}-05\end{array}$ & $\begin{array}{l}3.80 \mathrm{E}-06 \\
4.82 \mathrm{E}-06\end{array}$ & $\begin{array}{l}4.22 \mathrm{E}-07 \\
5.35 \mathrm{E}-07\end{array}$ \\
\hline $\begin{array}{l}\text { INTENTIONAL } \\
\text { HOSTILE ACTION }\end{array}$ & $\begin{array}{l}\text { Sabotage/ } \\
\text { War/Hostilities }\end{array}$ & General & $1.41 \mathrm{E}-05$ & 0.25 & 0.25 & 0.5 & $3.53 \mathrm{E}-06$ & $3.53 \mathrm{E}-06$ & 7.05E-06 \\
\hline $\begin{array}{l}\text { ACCIDENTAL / } \\
\text { INCIDENTAL } \\
\text { ACTION }\end{array}$ & $\begin{array}{l}\text { Impact due to } \\
\text { Farming / Excavation } \\
\text { / Pilfering }\end{array}$ & General & $1.55 \mathrm{E}-04$ & 0.5 & 0.5 & 0 & $7.75 \mathrm{E}-05$ & $7.75 \mathrm{E}-05$ & $0.00 \mathrm{E}+00$ \\
\hline $\begin{array}{l}\text { NATURAL } \\
\text { HAZARDS }\end{array}$ & & General & $1.41 \mathrm{E}-05$ & 0.1 & 0.2 & 0.7 & $1.41 \mathrm{E}-06$ & $2.82 \mathrm{E}-06$ & $9.87 \mathrm{E}-06$ \\
\hline TOTAL & & & & & & & $2.63 \mathrm{E}-04$ & $1.25 \mathrm{E}-04$ & 2.29E-05 \\
\hline
\end{tabular}

Table 8. Frequency values for segments, basic data and landslide.

\begin{tabular}{|c|c|c|c|c|c|c|c|}
\hline \multirow{3}{*}{ FEATURE } & \multirow{3}{*}{$\begin{array}{l}\text { KP } \\
(\mathbf{k m})\end{array}$} & \multicolumn{3}{|c|}{ FREQ FROM BASIC DATA } & \multicolumn{3}{|c|}{ FREQ FROM LANDSLIDE DATA } \\
\hline & & \multirow{2}{*}{ LEAK } & \multirow{2}{*}{ HOLE } & \multirow{2}{*}{ RUPTURE } & LEAK & HOLE & RUPTURE \\
\hline & & & & & 0.1 & 0.2 & 0.7 \\
\hline Land & 1 & 2.63E-04 & $1.25 \mathrm{E}-04$ & $2.29 \mathrm{E}-05$ & $1.48 \mathrm{E}-06$ & $2.96 \mathrm{E}-06$ & $1.04 \mathrm{E}-05$ \\
\hline Land & 2 & $2.63 \mathrm{E}-04$ & $1.25 \mathrm{E}-04$ & $2.29 \mathrm{E}-05$ & $8.49 \mathrm{E}-06$ & $1.70 \mathrm{E}-05$ & $5.94 \mathrm{E}-05$ \\
\hline Land & 3 & $2.63 \mathrm{E}-04$ & $1.25 \mathrm{E}-04$ & $2.29 \mathrm{E}-05$ & $4.71 \mathrm{E}-08$ & $9.43 \mathrm{E}-08$ & $3.30 \mathrm{E}-07$ \\
\hline Land & 5 & $2.63 \mathrm{E}-04$ & $1.25 \mathrm{E}-04$ & $2.29 \mathrm{E}-05$ & $2.42 \mathrm{E}-06$ & $4.84 \mathrm{E}-06$ & $1.69 \mathrm{E}-05$ \\
\hline Land & 6 & 2.63E-04 & $1.25 \mathrm{E}-04$ & $2.29 \mathrm{E}-05$ & $1.59 \mathrm{E}-06$ & $3.18 \mathrm{E}-06$ & $1.11 \mathrm{E}-05$ \\
\hline Land & 7 & 2.63E-04 & $1.25 \mathrm{E}-04$ & $2.29 \mathrm{E}-05$ & $1.91 \mathrm{E}-06$ & $3.81 \mathrm{E}-06$ & $1.33 \mathrm{E}-05$ \\
\hline Land & 8 & $2.63 \mathrm{E}-04$ & $1.25 \mathrm{E}-04$ & $2.29 \mathrm{E}-05$ & $1.67 \mathrm{E}-09$ & 3.33E-09 & $1.17 \mathrm{E}-08$ \\
\hline
\end{tabular}

Table 9. Frequency values for segments, seismic data and river hazards.

\begin{tabular}{|c|c|c|c|c|c|c|c|}
\hline \multirow{3}{*}{ FEATURE } & \multirow{3}{*}{$\begin{array}{l}\text { KP } \\
(\mathbf{k m})\end{array}$} & \multicolumn{3}{|c|}{ FREQ FROM SEISMIC DATA } & \multicolumn{3}{|c|}{ RIVER HAZARDS } \\
\hline & & LEAK & HOLE & RUPTURE & LEAK & HOLE & RUPTURE \\
\hline & & 0.1 & 0.2 & 0.7 & 0.1 & 0.2 & 0.7 \\
\hline Land & 0 & & & & & & \\
\hline Land & 1 & $0.00 \mathrm{E}+00$ & $0.00 \mathrm{E}+00$ & $0.00 \mathrm{E}+00$ & $0.00 \mathrm{E}+00$ & $0.00 \mathrm{E}+00$ & $0.00 \mathrm{E}+00$ \\
\hline Land & 2 & $0.00 \mathrm{E}+00$ & $0.00 \mathrm{E}+00$ & $0.00 \mathrm{E}+00$ & $0.00 \mathrm{E}+00$ & $0.00 \mathrm{E}+00$ & $0.00 \mathrm{E}+00$ \\
\hline Land & 3 & $0.00 \mathrm{E}+00$ & $0.00 \mathrm{E}+00$ & $0.00 \mathrm{E}+00$ & $0.00 \mathrm{E}+00$ & $0.00 \mathrm{E}+00$ & $0.00 \mathrm{E}+00$ \\
\hline Land & 4 & $0.00 \mathrm{E}+00$ & $0.00 \mathrm{E}+00$ & $0.00 \mathrm{E}+00$ & $0.00 \mathrm{E}+00$ & $0.00 \mathrm{E}+00$ & $0.00 \mathrm{E}+00$ \\
\hline Land & 5 & $0.00 \mathrm{E}+00$ & $0.00 \mathrm{E}+00$ & $0.00 \mathrm{E}+00$ & $0.00 \mathrm{E}+00$ & $0.00 \mathrm{E}+00$ & $0.00 \mathrm{E}+00$ \\
\hline Land & 6 & $0.00 \mathrm{E}+00$ & $0.00 \mathrm{E}+00$ & $0.00 \mathrm{E}+00$ & $0.00 \mathrm{E}+00$ & $0.00 \mathrm{E}+00$ & $0.00 \mathrm{E}+00$ \\
\hline Land & 7 & $0.00 \mathrm{E}+00$ & $0.00 \mathrm{E}+00$ & $0.00 \mathrm{E}+00$ & $0.00 \mathrm{E}+00$ & $0.00 \mathrm{E}+00$ & $0.00 \mathrm{E}+00$ \\
\hline Land & 8 & $0.00 \mathrm{E}+00$ & $0.00 \mathrm{E}+00$ & $0.00 \mathrm{E}+00$ & $0.00 \mathrm{E}+00$ & $0.00 \mathrm{E}+00$ & $0.00 \mathrm{E}+00$ \\
\hline
\end{tabular}


Table 10. Frequency values for segments, gas line hazards and block valve stations.

\begin{tabular}{|c|c|c|c|c|c|c|c|}
\hline \multirow{3}{*}{ FEATURE } & \multirow{3}{*}{$\begin{array}{l}\text { KP } \\
(\mathbf{k m})\end{array}$} & \multicolumn{3}{|c|}{ CROSSING GAS LINE HAZARDS } & \multicolumn{3}{|c|}{ BV STATIONS } \\
\hline & & LEAK & HOLE & RUPTURE & LEAK & HOLE & RUPTURE \\
\hline & & 0.1 & 0.2 & 0.7 & 0.1 & 0.2 & 0.7 \\
\hline Land & 0 & & & & & & \\
\hline Land & 1 & $0.00 \mathrm{E}+00$ & $0.00 \mathrm{E}+00$ & $0.00 \mathrm{E}+00$ & & & \\
\hline Land & 2 & $0.00 \mathrm{E}+00$ & $0.00 \mathrm{E}+00$ & $0.00 \mathrm{E}+00$ & & & \\
\hline Land & 3 & $0.00 \mathrm{E}+00$ & $0.00 \mathrm{E}+00$ & $0.00 \mathrm{E}+00$ & & & \\
\hline Land & 4 & $0.00 \mathrm{E}+00$ & $0.00 \mathrm{E}+00$ & $0.00 \mathrm{E}+00$ & & & \\
\hline Land & 5 & $0.00 \mathrm{E}+00$ & $0.00 \mathrm{E}+00$ & $0.00 \mathrm{E}+00$ & & & \\
\hline Land & 6 & $0.00 \mathrm{E}+00$ & $0.00 \mathrm{E}+00$ & $0.00 \mathrm{E}+00$ & & & \\
\hline Land & 7 & $0.00 \mathrm{E}+00$ & $0.00 \mathrm{E}+00$ & $0.00 \mathrm{E}+00$ & & & \\
\hline Land & 8 & $0.00 \mathrm{E}+00$ & $0.00 \mathrm{E}+00$ & $0.00 \mathrm{E}+00$ & & & \\
\hline
\end{tabular}

Table 11. Frequency values for segments, total leak frequencies and block adjacent pipeline impact.

\begin{tabular}{|c|c|c|c|c|c|c|c|}
\hline \multirow{2}{*}{ FEATURE } & \multirow{2}{*}{$\begin{array}{l}\text { KP } \\
(\mathbf{k m})\end{array}$} & \multicolumn{3}{|c|}{ TOTAL LEAK FREQUENCIES } & \multicolumn{3}{|c|}{ ADJACENT PIPELINE IMPACT } \\
\hline & & LEAK & HOLE & RUPTURE & LEAK & HOLE & RUPTURE \\
\hline Land & 0 & & & & & & \\
\hline Land & 1 & $2.65 \mathrm{E}-04$ & $1.28 \mathrm{E}-04$ & $3.33 \mathrm{E}-05$ & $0.00 \mathrm{E}+00$ & $0.00 \mathrm{E}+00$ & $0.00 \mathrm{E}+00$ \\
\hline Land & 2 & 2.72E-04 & $1.42 \mathrm{E}-04$ & $8.23 \mathrm{E}-05$ & $0.00 \mathrm{E}+00$ & $0.00 \mathrm{E}+00$ & $0.00 \mathrm{E}+00$ \\
\hline Land & 3 & 2.64E-04 & $1.25 \mathrm{E}-04$ & 2.33E-05 & $0.00 \mathrm{E}+00$ & $0.00 \mathrm{E}+00$ & $0.00 \mathrm{E}+00$ \\
\hline Land & 4 & $2.65 \mathrm{E}-04$ & $1.27 \mathrm{E}-04$ & $3.02 \mathrm{E}-05$ & $0.00 \mathrm{E}+00$ & $0.00 \mathrm{E}+00$ & $0.00 \mathrm{E}+00$ \\
\hline Land & 5 & $2.66 \mathrm{E}-04$ & $1.30 \mathrm{E}-04$ & $3.99 \mathrm{E}-05$ & $0.00 \mathrm{E}+00$ & $0.00 \mathrm{E}+00$ & $0.00 \mathrm{E}+00$ \\
\hline Land & 6 & $2.65 \mathrm{E}-04$ & $1.28 \mathrm{E}-04$ & $3.41 \mathrm{E}-05$ & $0.00 \mathrm{E}+00$ & $0.00 \mathrm{E}+00$ & $0.00 \mathrm{E}+00$ \\
\hline Land & 7 & $2.65 \mathrm{E}-04$ & $1.28 \mathrm{E}-04$ & $3.63 \mathrm{E}-05$ & $0.00 \mathrm{E}+00$ & $0.00 \mathrm{E}+00$ & $0.00 \mathrm{E}+00$ \\
\hline Land & 8 & 2.63E-04 & $1.25 \mathrm{E}-04$ & $2.30 \mathrm{E}-05$ & $0.00 \mathrm{E}+00$ & $0.00 \mathrm{E}+00$ & $0.00 \mathrm{E}+00$ \\
\hline
\end{tabular}

Table 12. Spill values from each leak, calculated pipe considering pipe's geometry and location of block valves location.

\begin{tabular}{llll}
\hline \multirow{2}{*}{$\begin{array}{l}\text { KP } \\
(\mathbf{k m})\end{array}$} & LEAK & HOLE & RUPTURE \\
\cline { 2 - 4 } & Leak Area $\left(\mathbf{c m}^{\mathbf{2}}\right)$ & Leak Area $\left(\mathbf{c m}^{\mathbf{2}}\right)$ & Leak Area $\left(\mathbf{c m}^{\mathbf{2}}\right)$ \\
\cline { 2 - 4 } & $\mathbf{0 . 2}$ & $\mathbf{2 0 . 0}$ & full bore \\
\cline { 2 - 4 } & Spill Time $(\mathbf{h})$ & Spill Time $(\mathbf{h})$ & Spill Time $(\mathbf{h})$ \\
\hline & 72 & 25.3 & 24 \\
\hline & & & \\
2 & 451.83 & 3533.80 & 5245.31 \\
3 & 432.61 & 2721.47 & 4465.33 \\
4 & 418.88 & 2751.77 & 4518.32 \\
5 & 421.10 & 2697.42 & 4460.66 \\
6 & 394.51 & 2062.35 & 3874.67 \\
7 & 336.57 & 1143.66 & 3026.92 \\
8 & 326.57 & 1033.34 & 2922.79 \\
\hline
\end{tabular}

The volume of the oil in each segment is presented by Table 12.Tables 13, 14 and 15 are presenting risk values of leak, hole and rupture with environmental risk, respectively and Table 16 total environmental risk. The methodology of calculating risk is in the following: Fifty percent of oil is being recovered and cost of recovering oil for $\mathrm{m} 3$ oil is, if;

\begin{tabular}{ll}
\hline $0 \leq$ Environmental Max Probability $<0.1$ & Cost $=\$ 1000$ \\
$0.1 \leq$ Environmental Max Probability $<0.4$ & Cost $=\$ 2000$ \\
$0.4 \leq$ Environmental Max Probability $<0.6$ & Cost $=\$ 5000$ \\
$0.6 \leq$ Environmental Max Probability $<0.8$ & Cost $=\$ 7500$ \\
$0.8 \leq$ Environmental Max Probability & Cost $=\$ 10000$. \\
\hline
\end{tabular}

These dollar values can be always adjusted depending on area and land conditions. Environmental risk as dollar value becomes,

Risk as Dollar Value $=$ Frequency $*$ Volume of the Oil * Percentage of recoverable Oil * Cost.

Total cleaning cost, which is the risk at a segment, can found for leak, hole and rupture. The sum of these costs becomes risk at every segment.

Table 13. Risk measure as dollar at every segment for leaks.

\begin{tabular}{|c|c|c|c|c|c|c|c|}
\hline feature & $\begin{array}{l}\text { kp } \\
(\mathbf{k m})\end{array}$ & $\begin{array}{l}\text { leak } \\
\text { frequency }\end{array}$ & $\begin{array}{l}\text { volume of oil spilt / } \\
\text { leaked with pumps off } \\
\text { \& BVs shut }\end{array}$ & $\begin{array}{l}\text { level of success of } \\
\text { clean-up (any size } \\
\text { spillage) }\end{array}$ & $\begin{array}{l}\text { environmental } \\
\text { sensitivity to oil } \\
\text { pollution }\end{array}$ & $\begin{array}{l}\text { cost of pollution of } \\
\text { environment }[\$ / \mathrm{cu} \\
\mathrm{m}]\end{array}$ & $\begin{array}{l}\text { risk of environmental } \\
\text { pollution / impact } \\
\text { [\$/y] }\end{array}$ \\
\hline $\begin{array}{l}\text { basic } \\
\text { dummy }\end{array}$ & & $2.00 \mathrm{e}-04$ & 100 & 0.5 & 0.50 & 5000 & 50.0 \\
\hline land & 0.0 & $0.00 \mathrm{e}+00$ & 0 & 0.5 & 0.000 & 1000 & 0.0 \\
\hline land & 1.0 & $2.65 \mathrm{e}-04$ & 452 & 0.5 & 0.750 & 7500 & 449.0 \\
\hline land & 2.0 & $2.72 \mathrm{e}-04$ & 433 & 0.5 & 0.750 & 7500 & 441.2 \\
\hline land & 3.0 & $2.64 \mathrm{e}-04$ & 419 & 0.5 & 0.750 & 7500 & 414.0 \\
\hline land & 4.0 & $2.65 \mathrm{e}-04$ & 421 & 0.5 & 0.750 & 7500 & 417.7 \\
\hline land & 5.0 & $2.66 \mathrm{e}-04$ & 395 & 0.5 & 0.750 & 7500 & 393.4 \\
\hline land & 6.0 & $2.65 \mathrm{e}-04$ & 337 & 0.5 & 0.750 & 7500 & 334.6 \\
\hline land & 7.0 & $2.65 \mathrm{e}-04$ & 327 & 0.5 & 0.750 & 7500 & 325.0 \\
\hline land & 8 & $2.63 e-04$ & 338 & 0.5 & 0.75 & 7500 & 334.5 \\
\hline
\end{tabular}


Table 14. Risk measure as dollar at every segment for holes.

\begin{tabular}{|c|c|c|c|c|c|c|c|}
\hline feature & $\begin{array}{l}\text { kp } \\
(\mathbf{k m})\end{array}$ & $\begin{array}{l}\text { leak } \\
\text { frequency }\end{array}$ & $\begin{array}{l}\text { volume of oil spilt / } \\
\text { leaked with pumps off } \\
\text { \& BVs shut }\end{array}$ & $\begin{array}{l}\text { level of success } \\
\text { of clean-up (any } \\
\text { size spillage) }\end{array}$ & $\begin{array}{l}\text { environmental } \\
\text { sensitivity to oil } \\
\text { pollution }\end{array}$ & $\begin{array}{l}\text { cost of pollution of } \\
\text { environment }[\$ / \mathrm{cu} \\
\mathrm{m}]\end{array}$ & $\begin{array}{l}\text { risk of environmental } \\
\text { pollution / impact } \\
\text { [\$/y] }\end{array}$ \\
\hline Land & 0 & $0.00 \mathrm{E}+00$ & 0 & 0.5 & 0.00 & 1000 & 0.0 \\
\hline Land & 1 & $1.28 \mathrm{E}-04$ & 3534 & 0.5 & 0.000 & 1000 & 225.5 \\
\hline Land & 2 & $1.42 \mathrm{E}-04$ & 2721 & 0.5 & 0.000 & 1000 & 192.7 \\
\hline Land & 3 & $1.25 \mathrm{E}-04$ & 2752 & 0.5 & 0.000 & 1000 & 171.7 \\
\hline Land & 4 & $1.27 \mathrm{E}-04$ & 2697 & 0.5 & 0.100 & 1000 & 170.9 \\
\hline Land & 5 & $1.30 \mathrm{E}-04$ & 2062 & 0.5 & 0.100 & 1000 & 133.5 \\
\hline Land & 6 & $1.28 \mathrm{E}-04$ & 1144 & 0.5 & 0.100 & 1000 & 73.1 \\
\hline Land & 7 & $1.28 \mathrm{E}-04$ & 1033 & 0.5 & 0.100 & 1000 & 66.4 \\
\hline Land & 8 & $1.25 \mathrm{E}-04$ & 4635 & 0.5 & 0.100 & 1000 & 288.9 \\
\hline
\end{tabular}

Table 15. Risk measure as dollar at every segment for ruptures.

\begin{tabular}{l|lllllll}
\hline feature & $\begin{array}{l}\mathbf{k p} \\
\mathbf{( k m )}\end{array}$ & $\begin{array}{l}\text { leak } \\
\text { frequency }\end{array}$ & $\begin{array}{l}\text { volume of oil spilt / } \\
\text { leaked with pumps } \\
\text { off \& BVs shut }\end{array}$ & $\begin{array}{l}\text { level of success of } \\
\text { clean-up (any size } \\
\text { spillage) }\end{array}$ & $\begin{array}{l}\text { environmental } \\
\text { sensitivity to oil } \\
\text { pollution }\end{array}$ & $\begin{array}{l}\text { cost of pollution } \\
\text { of environment } \\
\text { [\$/cu m] }\end{array}$ & $\begin{array}{l}\text { risk of environmental } \\
\text { pollution / impact } \\
\text { [\$/y] }\end{array}$ \\
\hline BASIC & & $2.00 \mathrm{E}-04$ & 100 & 0.5 & 0.50 & 5000 & 50.0 \\
DUMMY & & & 0.5 & 0.000 & 1000 & 0.0 \\
Land & 0 & $0.00 \mathrm{E}+00$ & 0 & 0.5 & 0.750 & 7500 & 655.1 \\
Land & 1 & $3.33 \mathrm{E}-05$ & 5245 & 0.5 & 0.750 & 7500 & $1,378.8$ \\
Land & 2 & $8.23 \mathrm{E}-05$ & 4465 & 0.5 & 0.750 & 7500 & 394.3 \\
Land & 3 & $2.33 \mathrm{E}-05$ & 4518 & 0.5 & 0.750 & 7500 & 504.8 \\
Land & 4 & $3.02 \mathrm{E}-05$ & 4461 & 0.5 & 0.750 & 7500 & 579.6 \\
Land & 5 & $3.99 \mathrm{E}-05$ & 3875 & 0.5 & 0.750 & 7500 & 386.7 \\
Land & 6 & $3.41 \mathrm{E}-05$ & 3027 & 0.5 & 0.750 & 7500 & 397.6 \\
Land & 7 & $3.63 \mathrm{E}-05$ & 2923 & 0.5 & 0.75 & 7500 & 562.52 \\
Land & 8 & $2.30 \mathrm{E}-05$ & 6535 & & & \\
\hline
\end{tabular}

Table 16. Total risk measure as dollar at every segment for leak, holes and ruptures.

\begin{tabular}{llllll}
\hline Environmental risk $[\mathbf{\$} / \mathbf{y}]$ & & & & \\
\hline \multirow{2}{*}{ FEATURE } & $\begin{array}{l}\text { KP } \\
(\mathbf{k m})\end{array}$ & $\begin{array}{l}\text { Pipeline } \\
\text { Leak }\end{array}$ & $\begin{array}{l}\text { Pipeline } \\
\text { Hole }\end{array}$ & $\begin{array}{l}\text { Pipeline } \\
\text { Rupture }\end{array}$ & $\begin{array}{l}\text { Total } \\
\text { Risk }\end{array}$ \\
\hline Land & 0 & 0.00 & 0.00 & 0.00 & 0.00 \\
Land & 1 & 449.0 & 1691.2 & 655.1 & $2,795.28$ \\
Land & 2 & 441.2 & 1445.5 & 1378.8 & $3,265.49$ \\
Land & 3 & 414.0 & 1287.4 & 394.3 & $2,095.66$ \\
Land & 4 & 417.7 & 1281.9 & 504.8 & $2,204.51$ \\
Land & 5 & 393.4 & 1001.6 & 579.6 & $1,974.56$ \\
Land & 6 & 334.6 & 548.3 & 386.7 & $1,269.57$ \\
Land & 7 & 325.0 & 497.8 & 397.6 & $1,220.46$ \\
Land & 8 & 334.5 & 2166.7 & 562.5 & $3,063.70$ \\
\hline
\end{tabular}

These values can be used for various statistical interpretations and graphical presentation. The following risks can be included more detailed risk analysis, [18],

- Risk to Company Operations [\$/Year].

- Probability Staff Exposed to Fatal Consequences of Explosion,

- Probability Public Exposed to Fatal Consequences of Fire at Leak Site,

- Probability Public Exposed to Fatal

- Consequences of Fire at Remote Ignition Site,

- Probability Public Exposed to Fatal

- Consequences of Explosion,

- Total Number of Staff Exposed to Fire,

- Total Number of Staff Exposed to Explosion,

- Total Number of Public Exposed to Fire,

- Total Number of Public Exposed to Explosion,

- Sensitivity of Environment to Effects of Fire / Explosion,
- Individual Risk to Staff [Fatality/Year],

- Individual Risk to General Public [Fatality/Year],

- Societal Risk to Staff [Fatalities/Year],

- Societal Risk to Public [Fatalities/Year],

- Total Societal Risk [Fatalities/Year],

- Cost of Life of Member of Staff [\$],

- Cost of Life of Member of Public [\$],

- Cost of Equipment Lost In Accident and Its Replacement [\$],

- Loss of Revenue Resulting From

- Accident [\$],

- Penalties to Company - Fines, Profit Loss, Adverse Publicity [\$]

- Risk to Company Operations [\$/Year].

\section{Conclusion}

The methodology presented is a detailed analysis of quantifying risk of crude oil pipelines. The methodology can be easily be used for any crude oil pipeline with local frequency values. These values may be improved through new statistical data. In considering risk valorization characteristics of pipeline transport system and operation, it has been defined as the process valorization risk. The values acceptable and tolerable can be defined.

This study of risk of pipeline transport system activity definition presented the individual risk and total risk estimation infrastructures based on the characteristics of the infrastructure. These evaluations are based on results of estimation threatening consequences of events identified. The flow of tables presents computational details. The procedures 
and methodology can be used without difficulty.

\section{References}

[1] Risk Assessment, American Institute of Chemical Engineers, New York, 1995.

[2] W. K. Muhlbauer, Pipeline Risk Management Manual, Second Edition, Gulf Publishing Co., Houston, TX, 1999.

[3] Bercha Engineering Limited. "Northern Gateway Pipeline Public Safety Quantitative Risk Analysis", Final Report (P1005.02) to Northern Gateway Project. May 2012.

[4] K. Muhlbauer, Pipeline Risk Management Manual, Third Edition, Gulf Publishing Co., Houston, TX, 2004

[5] Ramesh Singh, Pipeline Integrity Handbook: Risk Management and Evaluation Paperback, Gulf Professional Publishing, October 25, 2013.

[6] H. A. Kishawy and H. A. Gabbar, Review of pipeline integrity management practices, International Journal of Pressure Vessels and Piping, Volume 87, Issue 7, Pages 373-380, July 2010 .

[7] Center for Chemical Process Safety (CCPS), Guidelines for Chemical Process Quantitative Risk Analysis (CPQRA), American Institute of Chemical Engineers, New York, New York, 1989.

[8] Center for Chemical Process Safety (CCPS), Guidelines for Hazard Evaluation Procedures Analysis (CPQRA), American Institute of Chemical Engineers, New York, 1992.

[9] T. Gunton and Sean Broadbent, A Spill Risk Assessment of the Enbridge Northern Gateway Project School of Resource and Environmental Management Simon Fraser University, April 2013.
[10] CONCAWE, Performance of cross-country pipelines in Western Europe - statistical summary of reported spillages Report No. 7/97. Brussels: CONCAWE, 1997.

[11] CONCAWE, Performance of cross-country pipelines in Western Europe - statistical summary of reported spillages Report No. 7/97. Brussels: CONCAWE, 1997.

[12] CONCAWE Report No 2/98: Western European Cross Country Oil Pipelines 25 Year Performance Statistics, 1998.

[13] CONCAWE Report No 3/00: Performance Of Cross Country Oil Pipelines In Western Europe Statistical Summary Of Reported Spillages, 1999.

[14] C. Bersani, L. Citro, R. V. Gagliardi, R. Sacile and A. M. Tomasoni, Accident occurrance evaluation in the pipeline transport of dangerous goods, Chemical Engineering Transactions 19, 249-254, 2010.

[15] T. Breton, J. C. Sanchez-Gheno, J. L. Alamilla and J. Alvarez-Ramirez, Identification of failure type in corroded pipelines: A Bayesian probabilistic approach, Journal of hazardous materials, 179, no. 1, 628-634, 2010.

[16] Det Norske Veritas AS, Energy Report, Recommended Failure Rates for Pipelines' Report no/DNV Reg. No.: 2009-1115, Rev 1, 2010-11-16, 2010.

[17] Handbook Failure Frequencies 2009,For Drawing Up A Safety Report, Flemish Government, Lne Department Environment, Nature and Energy Policy Unit Safety Reporting Division, 05/05/2009.

[18] C. H. Achebe, U. C. Nneke, and O. E. Anisiji, Analysis of Oil Pipeline Failures in the Oil and Gas Industries in the Niger Delta Area of Nigeria, Proc. of the Intern. MultiConference of Engineers and Computer Scientists 2012, Vol. II, IMECS 2012, March 14-16, Hong Kong, 2012. 